\title{
3D-Visualisierung zur Eingabe von Präferenzen in Empfehlungssystemen
}

\author{
Johannes Kunkel, Benedikt Loepp, Jürgen Ziegler \\ Universität Duisburg-Essen
}

\section{Zusammenfassung}

In diesem Beitrag stellen wir ein interaktives Empfehlungssystem vor, bei dem Nutzer ihre Präferenzen in einer dreidimensionalen Visualisierung des Produktraums eingeben können. Die Darstellung in Form einer Landschaft spiegelt dabei das Profil des aktuellen Nutzers wider, und ermöglicht diesem sowohl in Kaltstartsituationen als auch bei der späteren Anpassung eines existierenden Profils interaktiv seine Präferenzen anzugeben. Die Methode basiert auf den von allen Nutzern abgegebenen Bewertungen und benötigt kein inhaltliches Wissen über die Produkte. Die durchgeführte Nutzerstudie zeigt, dass die Visualisierung nachvollziehbar und hilfreich erscheint. Bezüglich der Eingabe von Präferenzen durch Modellierung der Landschaft ergaben sich ebenfalls vielversprechende Ergebnisse, u. a. auch im Hinblick auf User Experience und Empfehlungsqualität.

\section{Einleitung}

Empfehlungssysteme (Recommender Systems) sollen durch proaktive Generierung von Vorschlägen Nutzer dabei unterstützen, aus sehr großen, meist unüberschaubaren Mengen von Items wie z. B. Filmen, Nachrichteninhalten oder Konsumgütern, diejenigen zu selektieren, die am besten zu ihren Interessen oder Präferenzen passen (Ricci et al. 2010). Erreichen die Systeme dabei eine gute Passung mit den Vorlieben des Nutzers, können sie den Interaktionsaufwand und die kognitive Belastung minimieren (Pu et al. 2011). Gängige Systeme nutzen dazu typischerweise Informationen über den Nutzer, die entweder implizit, z. B. durch Analyse des Browsing-Verhaltens, oder explizit, z. B. durch die Vergabe von Bewertungen, erhoben wurden (Ricci et al. 2010). Dabei mangelt es jedoch zumeist an Transparenz hinsichtlich der Ursachen für die gezeigten Empfehlungen (Tintarev \& Mastoff 2010). Außerdem haben Nutzer in der Regel keine oder nur begrenzte Möglichkeiten, die Empfehlungsgenerierung zu beeinflussen und Kontrolle auszuüben, obwohl sich gezeigt hat, dass Nutzer eine aktivere Rolle im Empfehlungsprozess wünschen (Xiao \& Bensabat 2007), und dafür sogar weniger präzise Empfehlungen sowie zusätzlichen Aufwand in Kauf nehmen (Konstan \& Riedl 2012). Zugleich existiert das Problem, Nutzer zu motivieren dem System ihre Präferenzen mitzuteilen, 
damit insbesondere neuen Nutzern in sogenannten Kaltstartsituationen möglichst schnell adäquate Vorschläge gemacht werden können. Auch können Nutzer aus Datenschutzgründen Bedenken haben, ihr Interessenprofil einem externen Anbieter preiszugeben. Es besteht deshalb Bedarf an Verfahren, die Nutzern eine intuitive, wenig aufwändige und transparente Angabe von Präferenzen ermöglichen.

Dieser Beitrag stellt ein interaktives Empfehlungssystem vor, bei dem Nutzer ihre Präferenzen in einer dreidimensionalen Visualisierung des Produktraums eingeben können. Zu diesem Zweck wird aus der Gesamtmenge vorhandener Nutzerbewertungen eine Anordnung der Items auf einer Ebene berechnet, bei der ohne Hinzunahme inhaltlichen Wissens die bewertungsbasierte Ähnlichkeit zwischen Items in räumliche Nähe abgebildet wird. Ist bereits ein Profil des aktuellen Nutzers vorhanden, werden dessen individuelle Präferenzen in Form von Bergen und Tälern in der dritten Dimension dargestellt. In einer Kaltstartsituation ist die Landschaft hingegen zunächst planar. In beiden Fällen kann die Landschaft dann mit Hilfe verschiedener Interaktionsmöglichkeiten verändert werden, um dem System die eigenen Präferenzen zu vermitteln. Nach solchen Änderungen am Präferenzprofil werden die Empfehlungen unmittelbar neu berechnet und angezeigt. Neben der Möglichkeit zur Einflussaufnahme auf den Empfehlungsprozess, hilft die Darstellung als Landschaft das Verständnis für das Zustandekommen der Vorschläge sowie die Nachvollziehbarkeit des im System hinterlegten Nutzerprofils zu verbessern.

\section{Visualisierungen in Empfehlungssystemen}

Lange Zeit war die Forschung zu Empfehlungssystemen auf die Verbesserung der Empfehlungsalgorithmen konzentriert. Weitere Optimierungen in dieser Hinsicht versprechen jedoch nur noch marginale Steigerungen der vom Nutzer wahrgenommenen Empfehlungsgüte (Pu et al. 2011). Zuletzt rückten deshalb nutzerzentrierte Fragestellungen stärker in den Fokus, z. B. hinsichtlich der Präsentation von Empfehlungen und der Interaktion mit den Systemen (Knijnenburg et al. 2012; Konstan \& Riedl 2012). Viele Empfehlungssysteme beschränken sich zur Eingabe von Präferenzen allerdings darauf, den Nutzer Items bewerten zu lassen z. B. mit Hilfe einer 5-stufigen, durch Sterne dargestellten Skala (Ricci et al. 2010). Diese Vergabe von Bewertungen erfolgt häufig losgelöst vom Empfehlungsprozess, so dass der Nutzer kein unmittelbares Feedback über die Auswirkungen seiner Aktionen erhält. Auch lassen sich meist nur einzelne Items bewerten, und damit nur diesen gegenüber die persönlichen Vorlieben ausdrücken. Tag-basierte Ansätze wie MovieTuner (Vig et al. 2011) gestatten dem Nutzer zwar auch in größerem Umfang Präferenzen anzugeben - gezielt Items aus einem ganzen Bereich des Produktraums auf- oder abzuwerten ist jedoch nicht möglich. Das interaktive Empfehlungssystem MyMovieMixer (Loepp et al. 2015) erlaubt dem Nutzer eine feingranulare Definition seiner Präferenzen gemäß unterschiedlicher Kriterien, benötigt hierfür aber ebenfalls inhaltliche Zusatzinformationen über die Items. Die resultierenden Empfehlungen werden unmittelbar angezeigt und verschiedene einfache Visualisierungen helfen zu verstehen, wie sich die Interaktion auswirkt und welche Kriterien in der Ergebnismenge erfüllt werden konnten. 
Im Bereich der Informationsvisualisierung (Heer et al. 2010), aber auch speziell im Information Retrieval (Hearst 2009), existieren verschiedenste Ansätze um große Datenmengen anschaulich aufzubereiten - etwa um das Browsing in Dokumentenkollektionen zu erleichtern. In Empfehlungssystemen ist die Unterstützung durch geeignete Visualisierungen hingegen bisher wenig ausgeprägt und beschränkt sich meist auf spezielle Zwecke, etwa um in Form mengenbasierter Diagramme die Herkunft generierter Empfehlungen in hybriden Systemen zu verdeutlichen (Verbert et al. 2014). Komplexere Visualisierungen erlauben mitunter auch die Exploration des gesamten Datensatzes, etwa durch Verwendung von Landkarten-Metaphern (Gansner et al. 2009). Solche kartenbasierten Darstellungen lassen sich mit verschiedenen Techniken erzeugen (Kagie et al. 2010), wobei Multidimensional Scaling besonders hervorzuheben ist. Bei diesem Verfahren werden Ähnlichkeiten zwischen Items derart in einen niedrigdimensionalen Raum abgebildet, dass die Entfernungen in der erzeugten Darstellung die Ähnlichkeiten der Items so genau wie möglich widerspiegeln. Auch Gansner et al. (2009) nennen diese Technik und stellen mit TV-Land ein Empfehlungssystem vor, das einen gesamten Produktraum von Fernsehsendungen visualisiert. Thematisch zusammenhängende Sendungen werden in der Karte durch Regionen, und die Bereiche, in denen die empfohlenen Items liegen, durch farbliche Markierungen hervorgehoben. Durch den Einsatz von Collaborative Filtering zur Empfehlungsgenerierung sind dazu ausschließlich Nutzerbewertungen und keine zusätzlichen Metadaten erforderlich. Gansner et al. verwenden hierzu die weit verbreitete Matrix Factorization (Koren et al. 2009). Während somit ein exploratives Vorgehen unterstützt wird, fehlt die Möglichkeit, das im System hinterlegte Profil des Nutzers anzuzeigen, so dass dieser es im Verhältnis zur gesamten Datenbasis oder den gezeigten Empfehlungen besser nachvollziehen könnte. Moin (2014) stellt Personalized Item Maps vor, welche sowohl die Position des Nutzers als auch die umliegenden Items im Produktraum visualisieren. Der Nutzer erlangt somit Einblick in sein Profil, allerdings ist dazu eine Anpassung der gängigen Matrix-FactorizationAlgorithmen notwendig und eine Evaluation z. B. durch Nutzerstudien fehlt bislang.

Ein wesentlicher Nachteil existierender Visualisierungen ist, dass sie zumeist nur Ausgaben darstellen und dem Nutzer keine oder nur eingeschränkte Interaktionsmöglichkeiten bieten, um etwa direkten Einfluss auf die Empfehlungsgenerierung zu nehmen. SmallWorlds (Gretarsson et al. 2010) ist ein Beispiel dafür, wie sich komplexe Visualisierungen verwenden lassen, um dem Nutzer nicht nur zu helfen, Beziehungen zwischen Items und dem eigenen Nutzerprofil zu verstehen, sondern diese auch zu manipulieren. Allerdings bietet der Ansatz keinen Überblick über die gesamte Datenbasis und ist auf bestimmte Daten (Graphen aus sozialen Netzen) angewiesen. Den vorgestellten Ansätzen ist zudem gemein, dass sie sich auf zweidimensionale Darstellungen beschränken. Des Weiteren können systemgenerierte Erklärungen der angezeigten Empfehlungen hilfreich sein, um deren Zustandekommen zu erläutern und die Transparenz der Systeme im Allgemeinen zu verbessern. Meist beschränkt sich ihr Einsatz jedoch auf textbasierte Varianten (Tintarev \& Masthoff 2010). Zusammenfassend lässt sich somit festhalten, dass die Generierung einer dreidimensionalen Visualisierung, die einerseits die Exploration der Datenbasis ermöglicht und zugleich das aktuelle Nutzerprofil verständlich darstellt, andererseits eine interaktive Eingabe der persönlichen Präferenzen über die Bewertung einzelner Items hinausgehend gestattet, ein vielversprechendes Ziel zu sein scheint. 


\section{3D-Visualisierung zur Eingabe von Präferenzen}

Um das im Folgenden beschriebene Konzept einer visuell-unterstützten Erstellung und Anpassung von Präferenzprofilen umzusetzen, wurde eine prototypische Web-Anwendung implementiert, die es Nutzern zum einen erlaubt das im Empfehlungssystem hinterlegte Nutzerprofil in visueller Form zu betrachten, zum anderen aber auch, es interaktiv anzupassen. Als Grundlage diente der MovieLens-10M-Datensatz ${ }^{1}$, der über 10 Mio. Bewertungen für etwa 10000 Filme abgegeben von ca. 72000 Nutzern enthält. Weder für die Positionierung der Items noch für die Darstellung des Nutzerprofils wurden weitere inhaltliche Daten hinzugezogen. Lediglich zur Anzeige zusätzlicher Informationen in der grafischen Oberfläche (z. B. Filmplakat, Erscheinungsjahr und Regisseur) wurden weitere Metadaten verwendet.

\subsection{Positionierung und Auswahl repräsentativer Items}

Um die Items der Datenbasis auf einer zweidimensionalen Karte abzubilden, werden drei Schritte durchlaufen (Abbildung 1, oben). Zunächst werden anhand der Bewertungen aller Nutzer Ähnlichkeiten zwischen den Items berechnet. Für diese Berechnung können prinzipiell verschiedene Verfahren genutzt werden. Wir verwenden Matrix Factorization (Koren et al. 2009), ein weit verbreitetes Collaborative-Filtering-Verfahren, welches demnach keine inhaltlichen Daten benötigt und zudem eine hohe Präzision aufweist: Aus sämtlichen Item-Bewertungen von allen im Datensatz vorhandenen Nutzern werden dabei automatisiert latente Faktoren abgeleitet, welche Nutzer bzw. Items gemäß mehr oder weniger offensichtlicher Eigenschaften charakterisieren. Die Ausprägung dieser Faktoren für jeden Nutzer und jedes Item liegt dann als Vektor vor. Die Vorhersage $\hat{r}_{u i}$, die ausdrückt, wie Nutzer $u$ das Item $i$ bewerten würde, wird über das Skalarprodukt des Nutzervektors $\vec{p}_{u}$ und des Item-Vektors $\vec{q}_{i}$ gebildet. Äquivalent dazu lassen sich auch die Vorhersagen $\hat{r}_{u}$ des Nutzers $u$ für sämtliche Items ermitteln (Multiplikation mit den Vektoren aller Items $Q$; Gleichung 1).

$$
\hat{r}_{u}=Q \cdot \vec{p}_{u}
$$

Um nun eine zweidimensionale, nutzerübergreifende Anordnung der Items zu generieren, wird deren Ähnlichkeit gemäß der latenten Faktoren bestimmt. Hierzu wird die euklidische Distanz zwischen den Item-Vektoren $\vec{q}_{i}$ berechnet. Die Ähnlichkeiten werden dann herangezogen, um mittels des bereits erwähnten Multidimensional Scaling (Kagie et al. 2010) Koordinaten für die Items zu erhalten. So wird sichergestellt, dass die Ähnlichkeiten zwischen den Items durch deren Entfernung in der resultierenden Darstellung möglichst akkurat abgebildet werden.

Prinzipiell könnte nun unmittelbar die Darstellung der Items in der Karte erfolgen, was jedoch aufgrund ihrer Anzahl (nach Bereinigung um wenig bewertete Items noch mehr als 3000) zu einer unübersichtlichen Darstellung führen würde. Als zusätzlicher Schritt zur Datenreduktion wird der Item-Raum daher mittels k-means-Clustering aufgeteilt. Aus jedem Cluster wird dann

1 http://grouplens.org/datasets/movielens/ 


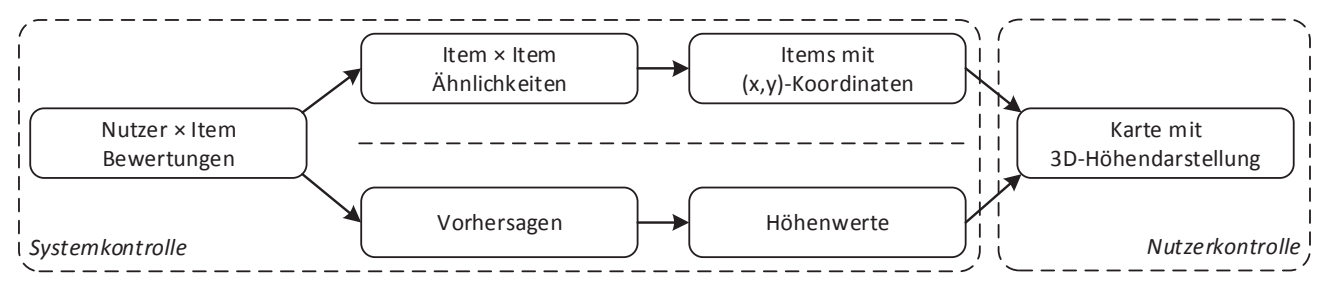

Abbildung 1: Schematische Darstellung des Datenflusses angelehnt an das Referenzmodell der Informationsvisualisierung (Card et al. 1999): Zum einen werden allgemeine Item-Ähnlichkeiten auf einer Karte dargestellt (oben), zum anderen die Präferenzen des aktuellen Nutzers in ein Höhenprofil transformiert (unten).

eines der populärsten Items gewählt, das exemplarisch als Repräsentant für diesen Bereich in der Karte angezeigt wird. Versuche mit Testpersonen zeigten, dass die Menge angezeigter Items so ca. um den Faktor 100 reduziert werden konnte, ohne ihre Aussagekraft zu reduzieren.

\subsection{Visualisierung von Nutzerprofil und Empfehlungen}

Die generierte zweidimensionale Karte, d.h., die Positionierung der Items, ist unabhängig vom betrachteten Nutzerprofil sondern spiegelt allgemeine Beziehungen zwischen den Items wider. Das Profil des aktuellen Nutzers, also seine Präferenzen, wirkt sich hingegen auf das Höhenprofil der Landschaft aus. Als Ausgangspunkt für die Berechnung dieses Höhenprofils dienen erneut ausschließlich Bewertungsdaten (Abbildung 1, unten): Wie in herkömmlichen Empfehlungssystemen werden anhand der vorhandenen Bewertungen des aktuellen Nutzers Vorhersagen für die noch nicht bewerteten Items approximiert, indem sein Nutzervektor $\vec{p}_{u}$ mit den Vektoren aller Items $Q$ multipliziert wird (vgl. Gleichung 1). Diese Bewertungsvorhersagen werden im Anschluss in Höhenwerte überführt, welche zusammen mit den Positionen aller Items zur Darstellung der Berge und Täler in der Landschaft genutzt werden. Somit wird eine Landschaft generiert, bei der die Positionierung der Items ihrer generellen Ähnlichkeit, das Höhenprofil jedoch den individuellen Präferenzen des aktuellen Nutzers entspricht. Zusätzlich zu den somit durch die Berge dargestellten allgemeinen Vorlieben des Nutzers (Bereiche, in denen für die Items hohe Bewertungen vorhergesagt wurden), werden auch die konkreten Topn-Empfehlungen in der Karte farbig umrandet und in einer Liste am unteren Bildschirmrand angezeigt (Abbildung 2).

\subsection{Interaktive Exploration und Präferenzmodellierung}

Um die generierte Landschaft interaktiv zu explorieren, bietet die Anwendung verschiedene Explorationswerkzeuge (Abbildung 2, oben links): Die Landschaft kann im virtuellen Raum rotiert, bewegt und hinein- bzw. hinausgezoomt werden. Sollten die repräsentativ dargestellten Items für eine Orientierung nicht ausreichen oder dem Nutzer unbekannt sein, ist es darüber hinaus möglich per Mausklick auf eine freie Fläche weitere, in dem jeweiligen Bereich positionierte Items anzuzeigen. Zugunsten der Übersicht können Items auch jederzeit wieder von der Karte entfernt werden. Inspiriert wurde dieses Verhalten von Stappers et al. (2000). 


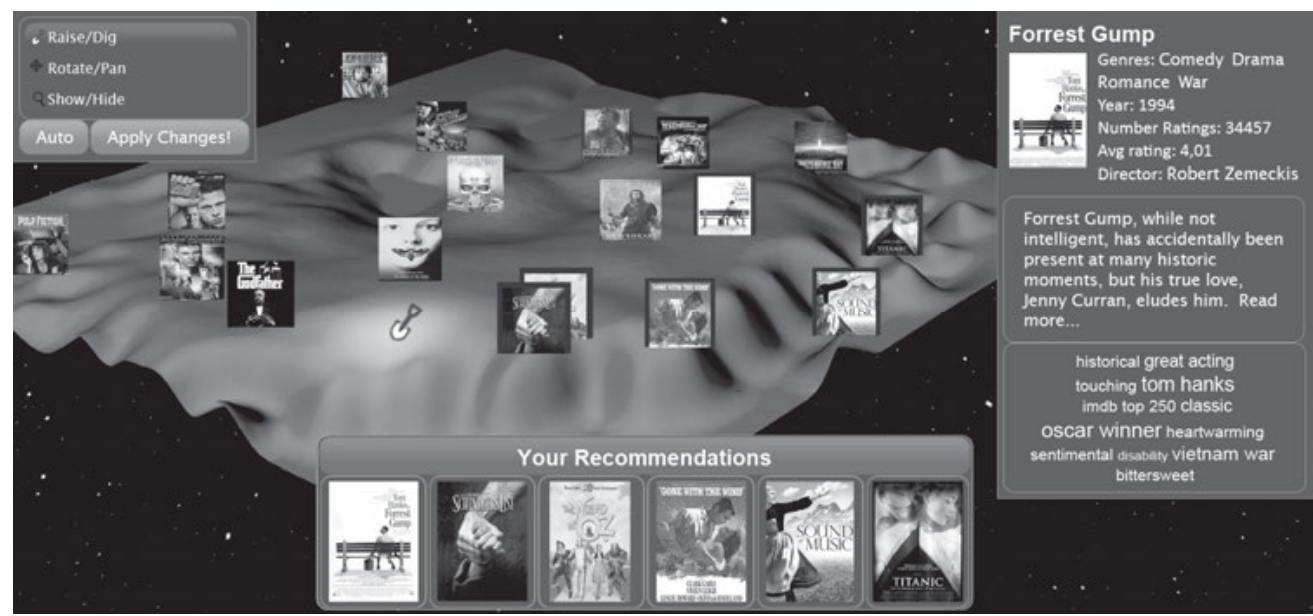

Abbildung 2: Für den Druck optimierter Screenshot, der das Profil eines Nutzers zeigt, das von den Höhen der Landschaft repräsentiert wird: Oben links kann u. a. das Modellierungswerkzeug selektiert werden, so dass sich per Maus (Schaufel) Berge und Täler formen lassen, um Präferenzen auszudrücken. Generierte Empfehlungen werden blau umrandet sowie in einer Liste (unten) dargestellt; rechts werden zudem Details eines gewählten Films gezeigt.

Neben diesen die Exploration von Datenbasis, Nutzerprofil und Empfehlungsmenge unterstützenden Möglichkeiten lässt sich außerdem mit einem Modellierungswerkzeug die Landschaft anpassen, um Einfluss auf das persönliche Präferenzprofil und damit unmittelbar auf die Empfehlung von potenziell interessanten Items nehmen zu können. Alternativ zu der Option ein existierendes Profil als Grundlage für weitere Anpassungen zu verwenden, kann in einer Kaltstartsituation mit einer flachen Landschaft begonnen werden (alle Höhenwerte initial auf neutralem Niveau). In beiden Fällen lässt sich nach Auswahl des Werkzeugs das Höhenprofil mit Hilfe der Maus modellieren. Indem der Nutzer Berge und Täler formt, kann er dem System mitteilen, welche Bereiche von Items er innerhalb des Produktraums bevorzugt oder eher weniger mag. Unabhängig davon, welche Items momentan auf der Karte dargestellt werden, wirkt sich diese Präferenzmodellierung dabei für den aktuellen Nutzer auf die gesamte Datenbasis aus: Durch die Modellierung verändert der Nutzer die zuvor für die Höhen verantwortlichen Vorhersagewerte $\hat{r}_{u}$ für sämtliche Items, was in neuen Präferenzwerten $x_{u}$ resultiert. Dies führt zu einer Anpassung seines in den Empfehlungsprozess eingehenden Nutzervektors $\vec{p}_{u}$. Algorithmisch wird hierzu Gleichung 1 mittels Singulärwertzerlegung nach $\vec{p}_{u}$ aufgelöst, um dann die gemäß des angepassten Höhenprofils veränderten Werte $x_{u}$ zur Neuberechnung des Nutzervektors heranzuziehen (Gleichung 2).

$$
\vec{p}_{u}=Q^{+} \cdot x_{u}
$$

Somit wird ein neuer Nutzervektor approximiert, der wiederum für die Empfehlungsgenerierung gemäß Gleichung 1 verwendet werden kann. Dies geschieht entweder explizit auf Wunsch des Nutzers (,Apply“-Button) oder bei aktiviertem „Auto-Modus“ kontinuierlich (vgl. Abbildung 2). Da sich durch die Empfehlungsgenerierung sämtliche Vorhersagewerte verändern, werden in Folge auch das Höhenprofil gemäß des in Abschnitt 3.2 gezeigten Vorgehens sowie die dargestellte Empfehlungsmenge aktualisiert. Die resultierende Anpassung der Landschaft ermöglicht dem Nutzer unmittelbar nachzuvollziehen, wie sich sein Profil durch seine getätigten Aktionen im System verändert. 


\section{Evaluation}

In einer Nutzerstudie wurde das System hinsichtlich seiner Eignung zur Steuerung der Empfehlungsgenerierung untersucht. Dabei standen die Fragen im Vordergrund, ob die generierte Landschaft verständlich und hilfreich ist, und ob deren Modellierung ein geeignetes Interaktionsmittel für den Nutzer darstellt, um seine Präferenzen anzugeben. Des Weiteren sollten die Faktoren User Experience und Empfehlungsqualität empirisch evaluiert werden.

\subsection{Studienaufbau und Datenerhebung}

Wir baten 32 Probanden (10 davon weiblich) mit einem Durchschnittsalter von 24.22 Jahren $(\sigma=3.61)$, die in einem gängigen Webbrowser laufende Anwendung im Rahmen einer Laborstudie zu nutzen und dabei folgende Aufgaben zu erledigen:

1. Exploration: Die Nutzer wurden gebeten mit Hilfe der Explorationswerkzeuge in maximal jeweils drei Minuten drei Filme in der Landschaft zu finden, die bestimmte Kriterien erfüllten: 1.1) populäre Filme (mehr als 30000 Bewertungen), 1.2) kinderfreundliche Filme, 1.3) Filme des Regisseurs Quentin Tarantino. Empfehlungen wurden nicht gezeigt.

2. Präferenzerhebung in Kaltstartsituationen: Die Probanden wurden gebeten, mit dem Modellierungswerkzeug eine flache Landschaft gemäß ihrer Präferenzen zu verformen, woraufhin vom System ein neues, diesen Eingaben entsprechendes Nutzerprofil generiert, und als neue Landschaft mit passenden Empfehlungen visualisiert wurde.

3. Anpassung bestehender Nutzerprofile: Den Probanden wurde jeweils eines von drei exemplarisch von uns ausgewählten, bestehenden Profilen aus dem Datensatz gezeigt, welches sie daraufhin in kontinuierlicher Interaktion so verändern sollten, dass die Empfehlungen eher dem eigenen Geschmack entsprachen.

Die Probanden wurden außerdem gebeten, einen Fragebogen auszufüllen: Vorab sollten Angaben zu Demografie, Domänenkenntnis und Nutzungsgewohnheiten hinsichtlich 3D-Anwendungen gemacht werden. Nach Aufgabe 2 und 3 sollten die Empfehlungsqualität, die Effizienz des Systems und die Transparenz der Empfehlungsgenerierung eingeschätzt werden (mit Hilfe von Items von Knijnenburg et al. (2012) und Pu et al. (2011)). Zusätzlich wurden selbstgenerierte Items zu den Möglichkeiten Kontrolle auszuüben, der Nutzungsabsicht und insbesondere zu den systemspezifischen Funktionalitäten (u. a. in Bezug auf Verständlichkeit der Landschaft und Nützlichkeit des Modellierungswerkzeugs) verwendet. Abschließend setzten wir den System Usability Scale (SUS; Brooke 1996) und den User Experience Questionnaire (UEQ; Laugwitz et al. 2008) ein. Um den generellen Nutzen des Systems, Motivation und Spaß bei dessen Verwendung sowie das Interesse der Nutzer zu evaluieren, verwendeten wir darüber hinaus Subskalen des Intrinsic Motivation Inventory (IMI; Ryan 1982). Bis auf bei UEQ (7-stufige bipolare Skala) und IMI (7-stufige Likert-Skala) wurden sämtliche Items auf einer positiven 5-stufigen Likert-Skala (1-5) erhoben. Mit Hilfe von Logdateien erfassten wir zudem das Interaktionsverhalten und die jeweilige Aufgabendauer. 


\subsection{Ergebnisse}

Die Versuchsteilnehmer gaben an, Filme zu mögen $(\mathrm{M}=3.91, \sigma=0.78)$ und kannten sich durchschnittlich gut mit Filmen aus $(\mathrm{M}=3.00, \sigma=0.95)$. 3D-Anwendungen wurden nur mäßig (Spiele: $\mathrm{M}=2.25, \sigma=1.41$ ) bis kaum (professionelle Werkzeuge: $\mathrm{M}=1.41, \sigma=0.61$ ) genutzt.

Bei der Explorationsaufgabe (Aufgabe 1) zeigte sich, dass das System wie erwartet besonders gut geeignet zu sein scheint, um Filme gemäß „weicher“ Kriterien zu finden. So konnte die Aufgabe, in drei Minuten drei Filme des Regisseurs Quentin Tarantino zu finden, nur von 56\% der Probanden erfolgreich absolviert werden. $88 \%$ der Probanden gelang es hingegen, besonders populäre Filme zu finden. Die Aufgabe, drei kinderfreundliche Filme zu finden, wurde von sämtlichen Probanden im Mittel in 1.12 Minuten $(\sigma=0.57)$ erfolgreich gelöst.

Tabelle 1 zeigt die nach Aufgabe 2 und 3 im weiteren Verlauf der Studie mit Hilfe von Fragebogen-Items ermittelten subjektiven Einschätzungen der Probanden.

\begin{tabular}{lcccc}
\hline & \multicolumn{2}{c}{ Aufgabe 2 } & \multicolumn{2}{c}{ Aufgabe 3 } \\
& M & $\sigma$ & M & $\sigma$ \\
\hline Empfehlungsqualität & 3.57 & 0.89 & 3.89 & 0.60 \\
Empfundener Aufwand & 3.75 & 0.76 & 3.21 & 0.93 \\
Kontrolle & 3.94 & 1.05 & 3.75 & 1.11 \\
Transparenz der Empfehlungen & 3.91 & 1.09 & 3.63 & 1.07 \\
Verständlichkeit der Landschaft & 3.94 & 0.91 & 3.41 & 1.04 \\
\hline
\end{tabular}

Tabelle 1: Subjektive Beurteilung der Empfehlungen und des Systems nach Aufgabe 2 und 3.

Unterschiede zwischen Aufgabe 2 und 3 zeigten sich bei Signifikanztests nur hinsichtlich des empfundenen Aufwandes $(t(31)=3.76, p<.01)$ und der Verständlichkeit der generierten Landschaften $(t(31)=2.37, p<.05)$. Aufgrund der unterschiedlichen Aufgabenstellung war dies jedoch zu erwarten: Auch wenn in Aufgabe 2 ein neues Profil aus der Landschaft modelliert werden sollte, war der mögliche Aufwand von vornherein begrenzt, da Empfehlungen erst hinterher angezeigt wurden (kein „Auto-Modus“). Da den Probanden in Aufgabe 3 zudem ein fremdes Profil gezeigt wurde, konnten diese erwartungsgemäß weniger gut nachvollziehen, wie die generierte Landschaft zustande kam. Insgesamt ergaben sich positive Resultate hinsichtlich der Empfehlungsqualität - in Kaltstartsituationen genauso wie bei Anpassung bestehender Profile. Während dabei die Möglichkeiten, Kontrolle auszuüben gut bewertet wurden, blieb der empfundene Aufwand akzeptabel, wobei die Ergebnisse (sowohl Empfehlungen als auch generierte Landschaften) verständlich erschienen. Die abschließenden Fragen bestätigten, dass die Visualisierung in Form einer Landschaft entscheidend hierzu beiträgt: Dieser wurde zugesprochen, dabei zu helfen, das Zustandekommen der Empfehlungen ( $\mathrm{M}=3.69$, $\sigma=0.93)$ und das im System hinterlegte Nutzerprofil $(M=3.63, \sigma=1.07)$ zu verstehen. Die Probanden fanden sich jederzeit gut in der Landschaft zurecht $(M=3.78, \sigma=1.01)$ und attestierten der Visualisierung hilfreich zu sein, um sich einen Überblick über die Datenbasis zu verschaffen $(M=3.91, \sigma=0.93)$. Besonders geeignet erschien den Probanden das System in Situationen, in denen keine $(M=3.91, \sigma=1.25)$ oder nur eine ungefähre $(M=4.06, \sigma=0.98)$ Suchrichtung vorhanden war. Mit einem konkreten Film vor Augen war die Nutzungsabsicht erwartungsgemäß deutlich geringer $(\mathrm{M}=2.44, \sigma=1.48)$. 
Insgesamt zeigten sich die Probanden zufrieden mit dem System $(M=3.94, \sigma=0.76)$ und bescheinigten ihm eine hohe Effektivität $(M=3.72, \sigma=0.74)$. Nennenswerte Zusammenhänge mit Domänenkenntnis oder Nutzung anderer 3D-Anwendungen ergaben sich nicht. Die Auswertung der Logdateien deutet allerdings darauf hin, dass mehr und längere Interaktion tendenziell zu weniger guten Resultaten führt. Während dies vermutlich damit zusammenhängt, dass das Modellierungswerkzeug für feingranulare Veränderungen weniger geeignet ist, bedarf dieser Aspekt noch weiterer Untersuchung. Unabhängig davon bestätigte die Auswertung des IMI, dass die Probanden das Empfehlungssystem als nützlich erachten ( $M=5.08, \sigma=1.26)$ - insbesondere hinsichtlich der Möglichkeit, diesem die eigenen Präferenzen interaktiv zu vermitteln. Bei dieser Tätigkeit empfanden die Probanden wiederum viel Spaß (M=5.46, $\sigma=0.97)$, so dass die interaktive Modellierung der Landschaft als ein geeignetes Mittel zur Steigerung des Engagements angesehen werden kann. Es erscheint somit möglich, die Nutzer zu vermehrten Eingaben zu motivieren, was besonders in Kaltstartsituationen relevant ist. Trotz teils komplexer Interaktionsmöglichkeiten wurde die Usability des Systems mit einem Score von 75 gemäß SUS als gut bewertet. Auch auf den Skalen des UEQ lagen die Werte (zwischen 0.84 und 2.10) in einem überwiegend als positiv, meist sogar gut bis sehr gut zu bewertenden Bereich. Die Skalen Durchschaubarkeit $(M=1.66)$, Stimulation $(M=1.56)$ und Originalität $(M=2.10)$ sind dabei hervorzuheben.

\section{Diskussion und Fazit}

Dieser Beitrag zeigt, wie sich ausschließlich auf Basis von Nutzerbewertungen mit gängigen Techniken wie Matrix Factorization und Multidimensional Scaling eine landschaftliche Darstellung des gesamten Produktraums für Empfehlungssysteme erzeugen lässt. Diese kann einerseits exploriert, andererseits mit Hilfe eines Modellierungswerkzeugs bearbeitet werden, um interaktiv Präferenzen einzugeben. Dies ermöglicht es, bei der Präferenzgewinnung von der bislang üblichen Bewertung einzelner Items zu einer Gesamtübersicht des Profils zu gelangen. Das System unterstützt sowohl Kaltstartsituationen als auch die Veränderung bestehender Nutzerprofile. Die durchgeführte Nutzerstudie bestätigt den hohen Spaßfaktor durch die eher spielerische Interaktion, so dass der Ansatz geeignet zu sein scheint - insbesondere wenn keine oder nur eine ungefähre Suchrichtung vorhanden ist - effektiv qualitativ gute Empfehlungen zu generieren und den Nutzer dabei zur Eingabe weiterer Präferenzdaten zu motivieren. Trotz der gesteigerten Möglichkeiten, den Empfehlungsprozess zu kontrollieren, wurde der Aufwand als akzeptabel empfunden. Bezüglich der generellen Transparenz, aber auch der Verständlichkeit der Visualisierung zeigten sich ebenfalls positive Ergebnisse - sei es hinsichtlich der Anordnung der Items, aber auch der Darstellung des Nutzerprofils und der generierten Empfehlungen. Insgesamt wurde der neuartige Ansatz für ein Empfehlungssystem mit dreidimensionaler Darstellung zur Eingabe von Präferenzen sehr positiv von den Probanden aufgenommen. Dennoch sind Verbesserungen z. B. hinsichtlich der Granularität des Modellierungswerkzeugs sowie der Höhendarstellung des Nutzerprofils denkbar. Dies, und die Optimierung der Empfehlungsalgorithmik, ist Gegenstand künftiger Arbeiten. Des Weiteren sind wir u. a. daran interessiert, die Untersuchung des Ansatzes in weiteren Domänen, ggf. auch domänenübergreifend, fortzuführen. 


\section{Literaturverzeichnis}

Brooke, J. (1996). SUS - A quick and dirty usability scale. Usability Evaluation in Industry, 189-194.

Card, S. K., Mackinlay, J. D. \& Shneiderman, B. (1999). Readings in information visualization: Using vision to think. Morgan Kaufmann.

Gansner, E., Hu, Y., Kobourov, S. \& Volinsky, C. (2009). Putting recommendations on the map: Visualizing clusters and relations. In Proc. RecSys '09. ACM. 345-348.

Gretarsson, B., O'Donovan, J., Bostandjiev, S., Hall, C. \& Höllerer, T. (2010). SmallWorlds: Visualizing social recommendations. Computer Graphics Forum, 29(3), 833-842.

Heer, J., Bostock, M. \& Ogievetsky, V. (2010). A tour through the visualization zoo. Communications of the ACM, 53(6), 59-67.

Hearst, M. A. (2009). Search User Interfaces. Cambridge University Press.

Kagie, M., van Wezel, M., \& Groenen, P. J. F. (2010). Map based visualization of product catalogs. In Recommender Systems Handbook. Springer. 547-576.

Knijnenburg, B. P., Willemsen, M. C., Gantner, Z., Soncu, H. \& Newell, C. (2012). Explaining the user experience of recommender systems. User Mod. and User-Adapted Interaction, 22(4-5), 441-504.

Konstan, J. A. \& Riedl, J. (2012). Recommender systems: From algorithms to user experience. User Mod. and User-Adapted Interaction, 22(1-2), 101-123.

Koren, Y., Bell, R. M. \& Volinsky, C. (2009). Matrix factorization techniques for recommender systems. IEEE Computer, 42(8), 30-37.

Laugwitz, B., Held, T. \& Schrepp, M. (2008). Construction and evaluation of a user experience questionnaire. In HCI and Usability for Education and Work. Springer. 63-76.

Loepp, B., Herrmanny, K. \& Ziegler, J. (2015). Blended recommending: Integrating interactive information filtering and algorithmic recommender techniques. In Proc. CHI '15. ACM. 975-984.

Moin, A. (2014). A unified approach to collaborative data visualization. In Proc. SAC '14. ACM.

Pu, P., Chen, L. \& Hu, R. (2011). A user-centric evaluation framework for recommender systems. In Proc. RecSys '11. ACM. 157-164.

Ricci, F., Rokach, L. \& Shapira, B. (Hrsg.). (2010). Recommender Systems Handbook. Springer.

Ryan, R. M. (1982). Control and information in the intrapersonal sphere: An extension of cognitive evaluation theory. Journal of Personality and Social Psychology, 43(3), 450-461.

Stappers, P. J., Pasman, G. \& Groenen, P. J. F. (2000). Exploring databases for taste or inspiration with interactive multi-dimensional scaling. In Proc. HFES '00. SAGE. 575-578.

Tintarev, N. \& Masthoff, J. (2010). Designing and evaluating explanations for recommender systems. In Recommender Systems Handbook. Springer. 479-510.

Verbert, K., Parra, D. \& Brusilovsky, P. (2014). The effect of different set-based visualizations on user exploration of recommendations. In Proc. IntRS '14.37-44.

Vig, J., Sen, S. \& Riedl, J. (2011). Navigating the tag genome. In Proc. IUI '11. ACM. 93-102.

Xiao, B. \& Benbasat, I. (2007). E-commerce product recommendation agents: Use, characteristics, and impact. MIS Quarterly, 31(1), 137-209. 